\title{
Instrumentation and Methodology for Quantifying GFP Fluorescence in Intact Plant Organs
}

\author{
R.J. Millwood, M.D. Halfhill ${ }^{1}$, D. Harkins ${ }^{2}$, R. Russotti ${ }^{2}$, and C.N. Stewart, Jr. \\ UNC Greensboro, Greensboro, NC, ${ }^{1} \mathrm{NC}$ State University, Raleigh, NC, and \\ ${ }^{2}$ Opti-Sciences, Tyngsboro, MA, USA
}

BioTechniques 34:638-643 (March 2003)

\begin{abstract}
The General Fluorescence Plant Meter (GFP-Meter) is a portable spectrofluorometer that utilizes a fiber-optic cable and a leaf clip to gather spectrofluorescence data. In contrast to traditional analytical systems, this instrument allows for the rapid detection and fluorescence measurement of proteins under field conditions with no damage to plant tissue. Here we discuss the methodology of gathering and standardizing spectrofluorescence data from tobacco and canola plants expressing GFP. Furthermore, we demonstrate the accuracy and effectiveness of the GFP-Meter. We first compared GFP fluorescence measurements taken by the GFP-Meter to those taken by a standard laboratory-based spectrofluorometer, the FluoroMax ${ }^{\circledR}-2$. Spectrofluorescence measurements were taken from the same location on intact leaves. When these measurements were tested by simple linear regression analysis, we found that there was a positive functional relationship between instruments. Finally, to exhibit that the GFP-Meter recorded accurate measurements over a span of time, we completed a time-course analysis of GFP fluorescence measurements. We found that only initial measurements were accurate; however, subsequent measurements could be used for qualitative purposes.
\end{abstract}

\section{INTRODUCTION}

Fluorescent proteins are becoming a staple technology in plant genetic research. GFP, from the jellyfish Aequorea victoria, was the first in this category of genetic markers to be cloned (1) and expressed in plant cells (2). It has proven to be a powerful tool in plant research because it is a universal, in vivo, and real-time transgenic marker (4-8). However, under field conditions, optimal detection and measurement of GFP in intact leaves and other plant organs have not occurred because of the lack of appropriate and affordable instrumenta- tion. This has limited researchers in their ability to collect data and poses a significant problem for future research.

There are several types of systems presently in use for the macroscopic detection and quantification of fluorescent compounds, including high-intensity UV lamps, spectrofluorometers (e.g., FluoroMax ${ }^{\circledR}$-2; Jobin Yvon \& Glen Spectra, Edison, NJ, USA), and scanning laser systems (e.g., FluorImager ${ }^{\mathrm{TM}}$; FluorImager SI, Molecular Dynamics, Sunnyvale, CA, USA). A hand-held 365-nm UV lamp, such as a UVP Model B 100 AP (UVP, Upland, CA, USA), allows for expeditious scanning of GFP in whole plants. However, the UV light must be used in darkness; it is only effective for UVexcitable GFP variants, and it cannot be used to quantify GFP fluorescence. Spectrofluorometers and fluorescence imaging systems are capable of detecting the presence of GFP and also allow for quantification of fluorescent tissues $(3,6,9,10)$. However, both systems are laboratory-based and expensive. For several years, plant researchers have sought a portable instrument that measures GFP in field plants under ambient lighting conditions. This paper describes such an instrument; the prototype General Fluorescence Plant Meter (GFP-Meter) (Opti-Sciences, Tyngsboro, MA, USA). We also describe procedures to perform in vivo fluorescence measurements using laboratory equipment as controls.

\section{MATERIALS AND METHODS}

\section{GFP-Meter}

The GFP-Meter is a self-contained, field-portable fluorescence detection and data logging instrument powered by an internal 1.2-ampere hour 12-V gel lead acid battery (Figure 1). A filtered light-emitting diode (LED) generates excitation 
light when powered on. A small portion of this light is monitored to compensate for temperature drift. The output of the LED is focused onto one of three fiber ports. A driver, controlled by a microprocessing unit (MPU), regulates the LED power level and compensates for changes in battery voltage. This excitation light travels through a bandpass filter to a fiber-optic cable and is then delivered to the sample. Attached to the end of this cable is a leaf clip, which has been installed to keep the cable in place. The light emitted from the sample enters back into the fiber-optic cable and is directed through a bandpass filter into a low noise preamplifier. This signal is then fed into an analog/digital signal processing and filtering unit slaved to the MPU. Fluorescence measurements appear in real time on a liquid crystal display in units of counts per second. A 12-selection keypad provides user management of test functions and setup. A nonvolatile memory chip (capable of storing 1020 sample points) assures that data will not be lost when power is turned off or the battery removed, and a RS-232 port enables downloading data to a computer. The GFP-Meter uses a modulated detection system to minimize the effects of temperature drift and stray light. Virtually any bandpass filter combination can be used for excitation and emission. For this study, a 465-nm filter with a bandwidth of $35 \mathrm{~nm}$ was used for excitation. Channel 1 (GFP channel) used a 530-nm filter with a bandwidth of $35 \mathrm{~nm}$ for emission, and channel 2 (chlorophyll channel) used a 680-nm filter with a bandwidth of $35 \mathrm{~nm}$ for emission. Chlorophyll data are not reported in this study.

\section{FluoroMax-2}

The FluoroMax-2 is a laboratory-based spectrofluorometer system that utilizes computer data processing. All FluoroMax-2 functions are under the total control of Datamax spectroscopy software (Galactic Industries, Salem, NH, USA). Light from a $150-\mathrm{W}$ xenon lamp enters an excitation spec-

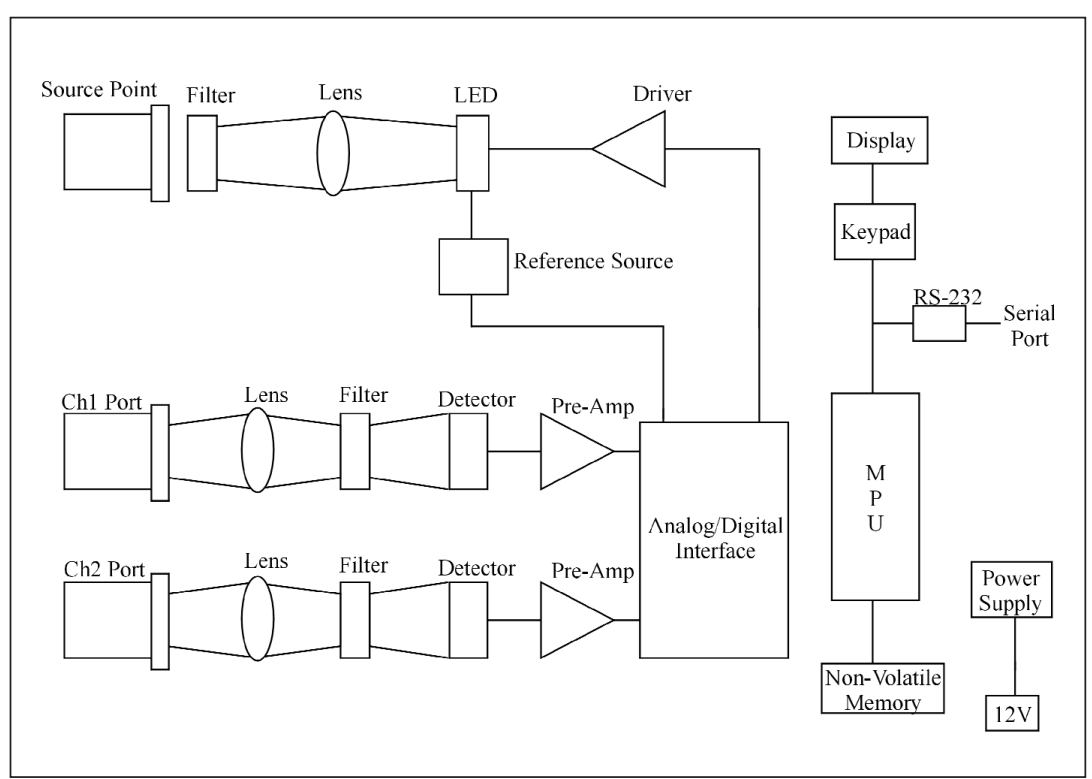

Figure 1. Technical schematic of the GFP-Meter. trometer, which delivers monochromatic light to a bifurcated fiber-optic cable. A rubber protector surrounds the external end of the cable and extends $4 \mathrm{~mm}$ beyond to prevent signal disruption from dirt and debris and to provide a dark environment for measurements. Light flows through the cable only when a sample is being scanned. When scanning, the cable is then placed onto the sample and light emitted from the sample flows back through the fiber-optic cable to the emission spectrometer where it is dispersed and directed to a signal photomultiplier detector. This fluorescence signal is then amplified and displayed on a computer monitor in units of counts per second.

\section{Plant Material}

Plants transgenic for GFP (m-gfp5-ER) (11) and GFP/Bt (m-gfp5-ER/Bacillus thuringiensis) under the control of constitutive $C a M V 35 S$ promoters were used in this study $(9,10)$. This GFP variant is excited equally by both blue $(465 \mathrm{~nm})$ and UV $(395 \mathrm{~nm})$ wavelengths. A total of $26 \mathrm{~T}_{1}$ canola (Brassica napus cv. Westar) plants were grown in a plant growth chamber (Percival Scientific, Perry, IA, USA). The photoperiod for the chamber was set at $14 / 10 \mathrm{~h}$ light/dark, and the temperature settings were $20^{\circ} \mathrm{C} / 17^{\circ} \mathrm{C}$ light/dark. Of these plants, there were eight lines expressing GFP and one control wild-type canola line (9). Plants were analyzed during the 12-15 leaf stage. Wild-type and GFP tobacco (Nicotiana tabacum cv. Xanthi) plants were grown in the greenhouse and field (10). The greenhouse (Greensboro, NC, USA) temperature ranged between $26^{\circ} \mathrm{C}$ and $30^{\circ} \mathrm{C}$, and the plants were exposed to ambient light from March to August. Field plants were grown in a fine sandy loam soil at the Upper Piedmont Experimental Station (Reidsville, NC, USA; 36:23 N and 79:42 W) from June to August 2001. The plants were germinated in the greenhouse and transplanted in the field 105 days after germination. Both greenhouse and field plants were analyzed 124 days after germination. Plants grown in their respective areas were germinated the same day and were grown under identical conditions.

\section{Fluorescent Spectrophotometry}

The GFP-Meter and the FluoroMax-2 spectrofluorometer were used to measure GFP fluorescence of intact leaves. A spot $19.6 \mathrm{~mm}^{2}$ on the underside of sample leaves and adjacent to the leaf mid-vein was excited at $465 \mathrm{~nm}$ with the GFP-Meter and $385 \mathrm{~nm}$ with the FluoroMax-2. GFP-Meter emission data were recorded at $530 \mathrm{~nm}$, and FluoroMax-2 emission spectra were recorded from 420 to $560 \mathrm{~nm}$.

The FluoroMax-2 GFP fluorescence scans were standardized to control samples to account for baseline variation of each leaf measurement. The protocol for standardization involves selection of a wavelength outside the GFP fluorescence spectrum as a point of 


\section{Product Application Focus}

normalization for each FluoroMax-2 scan. For this study, the 450-nm wavelength was the anchor point. Emissions spectra were recorded from four individual control plants and averaged. Subsequently, each emissions scan was standardized to the average control for that species. Functionally, the 450nm GFP value of the sample was subtracted from the 450 $\mathrm{nm}$ average control value. The resulting integer was then added to each wavelength value along the sample spectra (420-560 nm). This method eliminates differences outside the GFP emissions spectra, allowing for comparison of GFP magnitudes. The GFP-Meter did not have the option of standardization because the appropriate filter sets outside the GFP emissions range were not installed for this study.

\section{Time-Course Analysis}

A time-course study of GFP transgenic canola leaves was performed to analyze the stability and accuracy of GFP-Meter measurements over time, as compared with those of the FluoroMax-2. Measurements from both instruments were taken next to the mid-vein on the lower surface of a leaf. The fiber-optic cables used to transport excitation and emission light to and from the instruments were kept in the same position for the duration of the experiment. This was consistent for all samples. These measurements were recorded every 15 s over a 450 -s span. High- and lowexpressing GFP canola leaves were used in this study.

In this study, NIH and USDA guidelines were followed when working with recombinant DNA and transgenic plants.

\section{RESULTS AND DISCUSSION}

\section{Standardization}

Experimentation has demonstrated that plants from the same line exhibit baseline spectral differences when analyzed with FluoroMax-2 and excited with blue light (data not shown) and UV light (Figure 2). It is necessary to account for these differences so that the data are not misinterpreted. For example, field-grown GFP tobacco plants that have similar GFP synthesis, measured by Western blot analysis (data not shown), yield different GFP maximum values because of the baseline variation (Figure 2A). When the GFP spectra are standardized to the same $450-\mathrm{nm}$ value (Figure 2B), the fluorescence maxima at $510 \mathrm{~nm}$ are similar for both samples. In addition, when these GFP spectral scans were compared to high-expressing plants (Fig- ure 2, plants 4), it appears that the low-expressing plants (Figure 2, plants 3) are synthesizing equivalent amounts of GFP (Figure 2C). After standardization (Figure 2D), these apparently similar spectral scans are sorted according to their magnitude of GFP fluorescence. These data demonstrate that fluorescence scan standardization is necessary to obtain accurate measurements.

\section{Performance of GFP-Meter for Intact Leaf GFP Measurements}

Performance of the GFP-Meter was compared to the FluoroMax-2, a validated GFP fluorescence measurement tool $(6,9,10)$. A comparison of instantaneous measurements from the GFP-Meter (530-nm wavelength) and the FluoroMax-2 (standardized 510-nm wavelength fluorescence values) was completed to gauge accuracy. Even without the standardization capabilities built into the GFP-Meter, the regression analyses of growth chamber-grown GFP canola, greenhouse GFP tobacco, and field-grown GFP tobacco (Figure 3, A-C) produced high $\mathrm{R}^{2}$ values $(0.87,0.88$, and $0.89)$, which indicates a positive functional relationship between instruments. However, incorporating a standardization feature into the GFP-Meter may improve the instrument and produce higher $\mathrm{R}^{2}$ values.

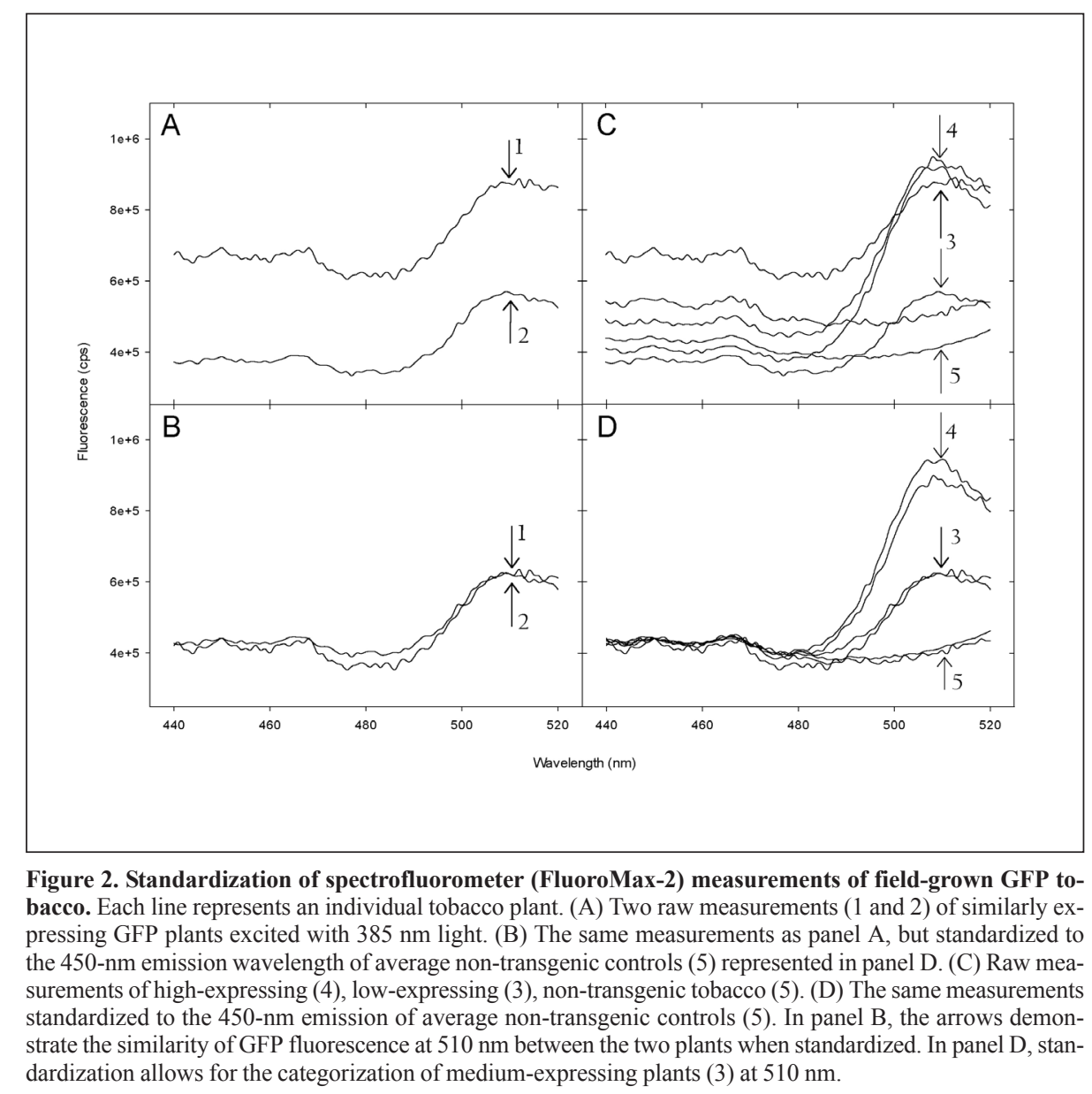

Figure 2. Standardization of spectrofluorometer (FluoroMax-2) measurements of field-grown GFP tobacco. Each line represents an individual tobacco plant. (A) Two raw measurements (1 and 2) of similarly expressing GFP plants excited with $385 \mathrm{~nm}$ light. (B) The same measurements as panel A, but standardized to the 450-nm emission wavelength of average non-transgenic controls (5) represented in panel D. (C) Raw measurements of high-expressing (4), low-expressing (3), non-transgenic tobacco (5). (D) The same measurements standardized to the 450-nm emission of average non-transgenic controls (5). In panel B, the arrows demonstrate the similarity of GFP fluorescence at $510 \mathrm{~nm}$ between the two plants when standardized. In panel D, standardization allows for the categorization of medium-expressing plants (3) at $510 \mathrm{~nm}$. 


\section{Product Application Focus}

\section{Time-Course Analysis}

Repeatability of the GFP-Meter was important to analyze to ensure that the instrument's readings were accurate over time. When we tested for the repeatability of the FluoroMax2 (repeated measurements, single spot on a leaf), we observed that there were no significant differences among measurements after standardization (data not shown). This was not the case with the GFP-Meter (Figure 4), where there was an obvious and unpredictable decrease in measurements over

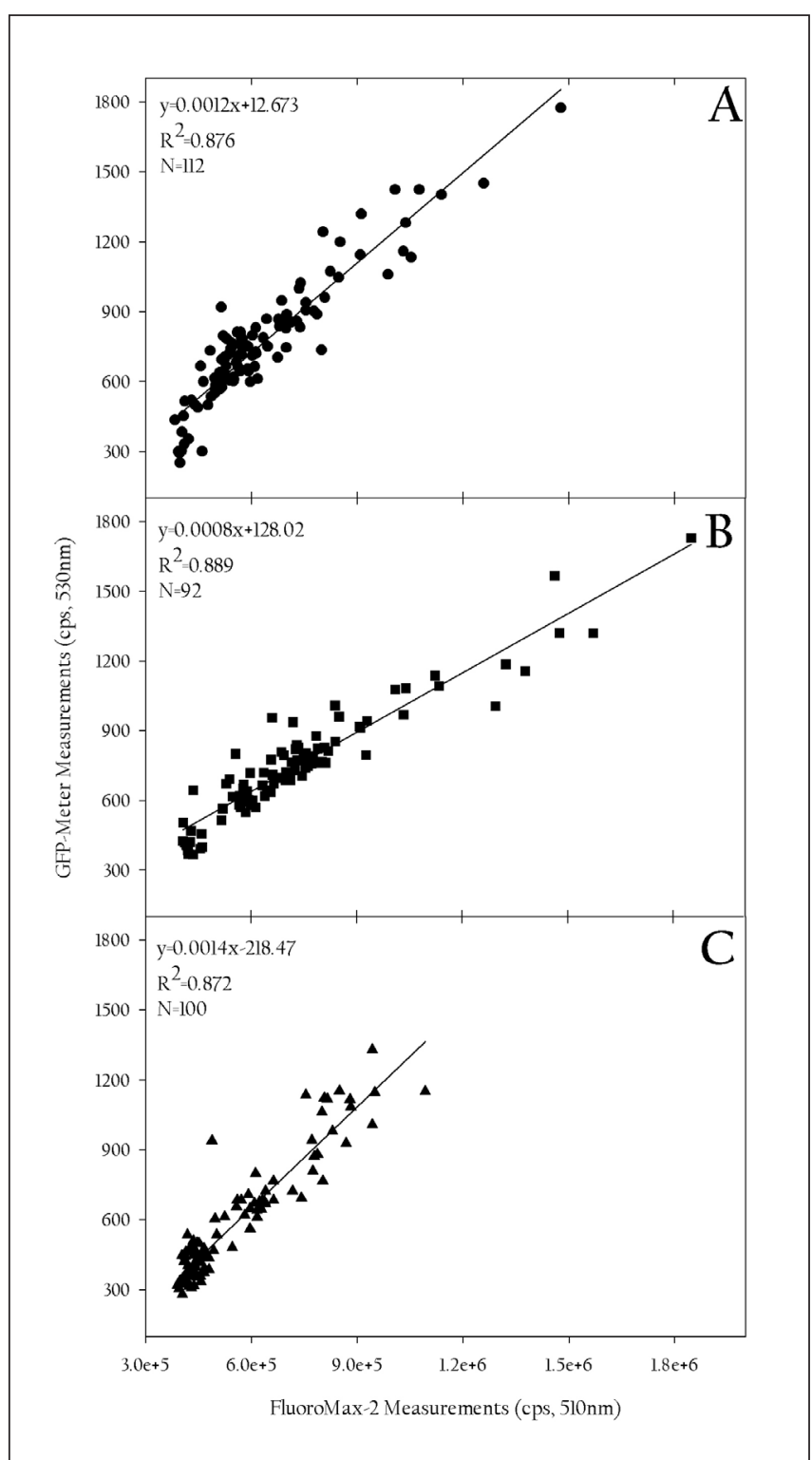

Figure 3. Comparisons of spectrofluorometer (FluoroMax-2) standardized measurements at the 510-nm wavelength and GFP-Meter measurements at the 530-nm wavelength. (A) GFP measurements of canola grown in an environmental control chamber. (B) GFP measurements of GFP tobacco grown in a greenhouse. (C) Measurements of GFP tobacco grown under field conditions (Reidsville, NC, USA). All measurements were recorded on intact or collected leaf tissues, and simple linear regression was performed to compare the measurements of both instruments. the course of time. After initial measurements, canola leaf sample fluorescence values demonstrated a sharp decline for the first $100 \mathrm{~s}$ but stabilized for the duration of the experiment. The same was true for tobacco (data not shown). This rate of decline was not consistent for each sample (Figure 4). Therefore, after the initial measurement, any measurements taken thereafter from the same location are incomparable for quantitative purposes.

\section{Optimizing the GFP-Meter for Use with GFP}

There are two practical concerns that should be noted about the GFP-Meter. The first concern involves baseline variation. Previously noted was that the FluoroMax-2 system experienced baseline variation among plants. In some cases, the FluoroMax-2 registered a 510-nm emission value for nontransformed plants that is similar to 510-nm fluorescence values for a plant expressing GFP (Figure 2); therefore, standardization is required. Consequently, if these values are not standardized, then a control plant may be mistaken as a plant expressing GFP. This baseline variation may be attributed to cable angle deviation from perpendicular when taking measurements with the FluoroMax-2; the user imprecisely controls the angle at which the fiber-optic cable is held. The angle at which the cable is held may influence the spectral scans, causing them to migrate on the y-axis at the scan start point (Figure 2, A and C). This may help to explain why the nonstandardized GFP-Meter data exhibited such strong correlations to the standardized FluoroMax-2 data. The GFP-Meter utilizes a leaf clip, which removes any chance of user variation because the fiber-optic cable is held at a fixed angle. However, since it is not known if the GFP-Meter exhibits some baseline variation, the instrument may be improved by adding a standardization feature. More research is being performed to determine baseline variation.

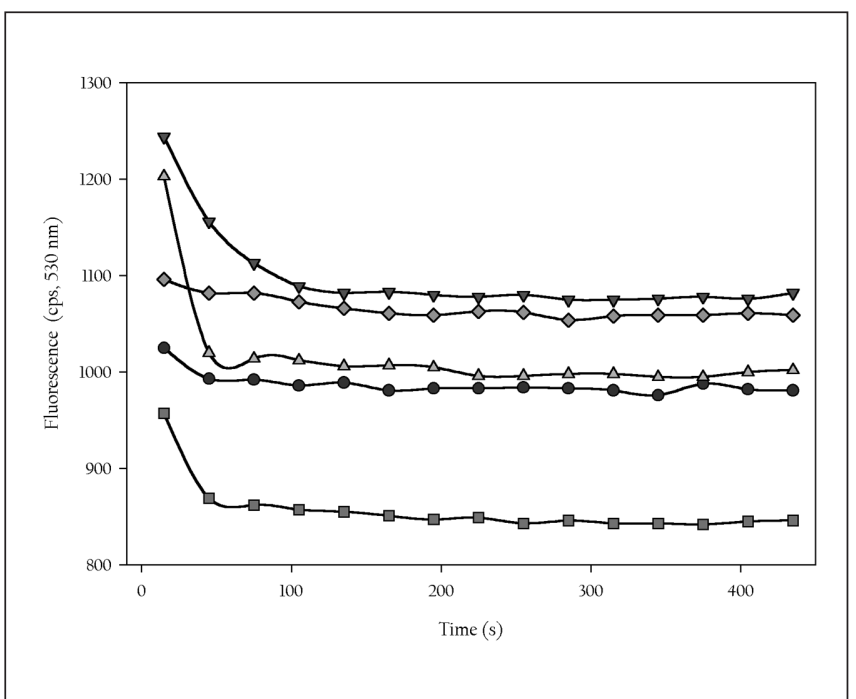

Figure 4. Time-course studies using the GFP-Meter. Each line represents an individual canola plant. The GFP-Meter leaf clip was placed at the same location on a single intact leaf, and GFP fluorescence measurements $(530-\mathrm{nm}$ wavelength) were recorded every $13 \mathrm{~s}$. 
The second concern involves comparing GFP-Meter fluorescence values when the measurements are taken after the leaf clip has been on the sample for more than $15 \mathrm{~s}$. Expression levels are incomparable if a reading is taken after this time because of an unpredictable decrease in fluorescence values (Figure 4). This decrease may be due to photobleaching of GFP, but minimal photobleaching would occur if measurements were taken immediately. It is also possible that the modulation source is inducing kinetics, as the graphed data points bore a distinct resemblance to a Kautsky curve (12); upon illumination of the plant material, there is a rapid rise in light emission from photosystem II fluorescence, followed by a decline as it gradually settles out to a low steady state. Although expression levels cannot be directly compared, the GFP-Meter can still be used for qualitative fluorescent protein detection purposes at any time.

Although these issues raise concerns about the effectiveness of the GFP-Meter, they can be adequately addressed. In addition to the normal operating procedures outlined in the usage manual (provided by Opti-Sciences), the following methods should be performed to ensure the accuracy of GFPMeter measurements. An emission filter, outside the GFP fluorescence range, should be installed in channel 2 of the GFP-Meter to control possible baseline variation. Channel 2 would then provide a value to be used for standardization to a control measurement. Furthermore, to minimize the effect of the decrease in fluorescence values over time, analysis should begin as soon as the leaf clip is placed onto the sample tissue. If these methods are followed, then GFP expression levels can be compared between individual plants and among plant lines.

In summary, the GFP-Meter was shown to be an effective tool for measuring the magnitude of GFP fluorescence in whole plant systems. Therefore, this instrument will allow researchers to gather data expeditiously and accurately. Additionally, there are other benefits to the GFP-Meter, such as nondestructive plant tissue analysis. This manner of data collection allows for other ecological and physiological analysis to be performed on the transgenic material. Another benefit of this instrument is its capability of employing different filter sets. This is encouraging, considering that fluorescent proteins from other marine organisms have recently been cloned (13). If these fluorescent proteins are incorporated in plant genetic research the same way GFP has been, then the potential utility of the GFP-Meter will increase. Furthermore, as the use of fluorescent proteins in biological applications increases, so should the applications of the GFP-Meter as a fluorescence detection and quantitation device.

\section{ACKNOWLEDGMENTS}

We thank Jim Haseloff for the gift of the m-GFP5-ER construct. We also would like to thank Harry Richards and Laura Hudson for their assistance. Grant funding for this work was from DARPA (5ER 544/105) and the USDA (2001-03726 and 00-34428-8987) to C.N.S.

\section{REFERENCES}

1.Prasher, D.C., V.K. Eckenrode, W.W. Ward, F.G. Prendergast, and M.J. Cormier. 1992. Primary structure of the Aequorea victoria green fluorescent protein. Gene 111:229-233.

2.Niedz, R.P., M.R. Sussman, and J.S. Satterlee. 1995. Green fluorescent protein: an in vivo reporter of plant gene expression. Plant Cell Rep. 14:403-406.

3.Niwa, Y., T. Hirano, K. Yoshimoto, M. Shimizu, and H. Kobayashi. 1999. Non-invasive quantitative detection and applications of non-toxic, S65T-type green fluorescent protein in living plants. Plant J. 18:455-463. 4.Haseloff, J. and B. Amos. 1995. GFP in plants. TIG 11:328-329.

5.Haseloff, J. and K.R. Siemering. 1998. The uses of GFP in plants, p. 191-220. In M. Chalfie and S.R. Kain (Eds.), Green Fluorescent Protein: Properties, Applications, and Protocols. Wiley and Sons, Chichester, UK.

6.Leffel, S.M., S.A. Mabon, and C.N. Stewart, Jr. 1997. Applications of green fluorescent protein in plants. BioTechniques 23:912-918.

7.Prasher, D.C. 1995. Using GFP to see the light. TIG 11:320-323.

8.Stewart C.N., Jr. 2001. The utility of green fluorescent protein in transgenic plants. Plant Cell Rep. 20:376-382

9.Halfhill, M.D., H.A. Richards, S.A. Mabon, and C.N. Stewart, Jr. 2001. Expression of Bt transgenes in Brassica napus and hybridization with Brassica rapa. Theor. Appl. Genet. 130:659-667.

10.Harper, B.K., S.A. Mabon, S.M. Leffel, M.D. Halfhill, H.A. Richards, K.A. Moyer, and C.N. Stewart, Jr. 1999. Green fluorescent protein as a marker for expression of a second gene in transgenic plants. Nat. Biotechnol. 17:1125-1129.

11.Haseloff, J., K.R. Siemering, D.C. Prasher, and S. Hodge. 1997. Removal of a cryptic intron and subcellular localization of green fluorescent protein are required to mark transgenic Arabidopsis plants brightly. Proc. Natl. Acad. Sci. USA 94:2122-2127.

12.Kautsky, H. and A. Hirsch. 1931. Neue Versuche zur Kohlensäureassimilation. Naturwissenschaften. 19:964.

13.Matz, M.V., A.F. Fradkov, Y.A. Labas, A.P. Savitsky, A.G. Zaraisky, M.L. Markelov, and S.A. Lukyanov. 1999. Fluorescent proteins from nonbioluminescent Anthozoa species. Nat. Biotechnol. 17:969-973.

Address correspondence to Dr. C. Neal Stewart, Jr., UT Knoxville, PSLS Department, 2431 Center Dr., 252 Ellington Building, Knoxville,TN 37996,USA.e-mail: nealstewart@ utk.edu 